\title{
An Investigation of Fucoidan as a Potential Inhibitor Against DENV/NS3 Proteases Through Molecular Dynamics Simulations and DFT Studies
}

\section{Ramalingam Swaminathan}

Mohamed sathak college of Arts and Science

Sankar Muthumanickam

Alagappa University

Venkataraman Anuradha

mohamed sathak college of Arts and Science

Mohamed yacoob Syed Ali

mohamed sathak College of Arts and Science

Boomi Pandi ( $\nabla$ pboomi1983@gmail.com )

Alagappa University https://orcid.org/0000-0001-8332-5596

Balajee Ramachandran

Alagappa University

\section{Research Article}

Keywords: ADME Prediction, Binding Free Energy, Fucoidan, Molecular Docking, Molecular Dynamics simulation, NS3 Proteases

Posted Date: February 22nd, 2022

DOl: https://doi.org/10.21203/rs.3.rs-1335554/v1

License: (c) (1) This work is licensed under a Creative Commons Attribution 4.0 International License. Read Full License 


\section{Abstract}

Dengue virus (DENV) is a mosquito-borne disease that spreads in the tropics and subtropics mainly by the Aedes aegypti mosquito, infecting more than 100 million people and cause copious deaths every year in globally. As of now, no direct-acting antiviral drugs or vaccine are available to combat DENV. Therefore, identification of novel small molecules from natural origin becomes inevitable for the management of DENV fever. Here, in silicofromPadinagymnospora was analyzed for competitive binding on the active site nonstructural protein NS3 proteases were selected that are potential therapeutic target for direct-acting antiviral. The fucoidan compound was docked with active site of NS3 proteases, showing good docking score and strong interaction with the catalytic dyad His51, Asp75 and Ser135 amino acids. Furthermore, Binding Free Energy was done by using prime MM/GBSA to access the binding affinity of fucoidan towards the target protein. Moreover, Molecular Dynamic Simulation was performed to evaluate the structural stability of the docked complexes. In addition, HOMO and LUMO for fucoidan phytocompound was done by DFT calculation, reveals the good chemical stability based on the energy value. The fucoidan phytocompound has satisfied all the relevant pharmacokinetic properties and also highly absorbed by the gastrointestinal tract. From the overall findings of this study, it is concluded that the fucoidan from Padinagymnospora has effectively blocked the catalytic dyad of NS3 proteases which could be considered as a potent inhibitor to control the DENV multiplication infection. Insilico approach plays vital role in the initial screening of lead molecules.

\section{Introduction}

Dengue Virus (DENV) belongs to the family Flaviviridae is an important mosquito-borne viral disease, one of the dangerous and fastest growing diseases especially amongst tropics and subtropical regions of the world. The disease highly contagious in India is spread by the Aedes aegypti mosquito [1]. It is reported that, more than 390 million people worldwide are affected each year including children [2]. The clinical symptoms for DENV include body fever up to $40^{\circ} \mathrm{C}$, severe muscle and joint pain, acute headache, skin rashes etc. After becoming ill, the fever does not appear immediately; it begins to appear 5 to 8 days after infection in patients [3]. The patients are continuously taking variety of drugs; however they are not effective and create a several side effects. This shows that there are severe urge drugs from natural source for treating this fatal disease. Several researchers are working day by day to come up with suitable alternative drug or medicine to control this deadly disease. However, an effective drug has not yet been discovered.

The DENV genome is made up of $11-\mathrm{kb}$ positive single-stranded RNA, which is then encodedsingle polyprotein precursor that is processed by cellular and viral proteases into three structural proteins (membrane, capsid and envelope) and seven non-structural proteins (NS1, NS2A, NS2B, NS3, NS4A, NS4B and NS5) [4]. The NS3 proteins are directly involved in the viral replication and assembly. It is highly conserved which consists of two functional domains such as serine protease (NS3 protease) located at the N-terminus and an RNA helicase (NS3hel) positioned at the C-terminus [5]. The NS3protease domain is an essential element of NS2B co-factor for their catalytic action, which helps to enhance the activity of NS3, participated in the substrate recognition and also serves as a central role for the assembly of the replication complex of DENV [6]. The viral NS2B/NS3 protease complex is mainly involved in the cleaving viral polyprotein precursor to 
forms a structural and nonstructural protein and to disrupt of NS2B/NS3 protease function as well as inhibitsthe viral replication and assembly [7].The nonstructural protein NS3 protease has been a well established therapeutic target for the development of antiviral agents against DENV infection [8]. In the field of drug discovery, natural bioactive compounds are efficient molecules without showing any toxicity effects which used to control and lessen the number of infections [9]. In particular, fucoidan is an essential sulfated polysaccharide phytocompound which is found in brown algae (Padinagymnospora). The fucoidan exhibits powerful biologicalactivities such as anti-viral, anti-coagulant, anti-tumor and anti-inflammatory due to possessing various chemical structures and compositions [10]. The in vitro antiviral activity results exhibited that the fucoidan phytocompound has reduced $48 \%$ viral infection and decreased $\mathrm{HN}$ protein expression byblocking the interaction of viruses to the host cells[11]. Hayashi et al. reported that the fucoidan molecule was isolated from Undariapinnatifida, an edible brown algae that exerts anti-IAV activity in mice [12]. Synytsya and co-workers reported that the fucoidan phytocompoundinhibit avian IAV (Influenza A virus) replication via enhancing the immune system in mice [12].

In the present study, fucoidan phytocompounds was selected based on their in vitrobiological activity tested against various diseases. Following that, fucoidan phytocompound was docked against DENVNS3proteases, then,Docking Score, Molecular interactions, Complex Stability, Binding Free Energy, Electronic Properties, Pharmaceutical relevant parameters and passive gastrointestinal absorption and brain access were examined through the in silico approach.

\section{Materials And Methods}

\section{Protein and ligand Preparation}

The three dimensional coordinate structures of DENV NS3 proteases were retrieved from the Protein Data Bank (PDB ID: 2FOM, 3U1Jand 2VBC for dengue virus 2, 3 and 4, respectively). All the protein structures were prepared by therequired essential steps followed by hydrogen atoms added and missing side chains (Schrödinger LLC, NY, USA). Following that, all the water molecules and co-crystal ligands were removed from the protein structures. Furthermore, energy minimization and optimization were done by using the OPLS2005 force field [13]. The fucoidan phytocompound was obtained from Pubchem database and it was prepared by the LigPrep module (Schrödinger LLC, NY, USA). Then, the structure was subjected to energy minimization with the OPLS2005 force field to remove the steric clashes of bond length,bond angles and it was maintained at $\mathrm{pH} 7 \pm 2[14]$.

\section{Molecular Docking and Binding Free Energy}

The Glide-XP module (Schrodinger LLC, NY, USA) was employed to perform molecular docking analysis with the binding site of NS3 protease proteins (DENV-2, DENV-3 and DENV-4) and to predicted the binding orientation of the fucoidan. The binding modes of these complexes were analyzed by using the Schrodinger package [15].The Schrödinger Prime MM-GBSA module (Schrödinger LLC, NY, USA) was applied to predict the binding Free Energy of fucoidan withDENV NS3 protease complexes [16]. The total binding free energy was estimated by using the following equation; 


\section{Molecular Dynamic Simulation}

The fucoidan phytocompound with acceptable docking scores were subjected to Molecular Dynamic Simulation to evaluate their structural flexibility and binding stability using GROMACS software [17]. The topology and parameter files for the protein-ligand molecules were generated by using GROMOS force field and PRODRG server [18]. Then, the docked complexes were solvated in a cubic box 1.0nm using Single Point Charge (SPC) water model followed by the system neutralized by adding $\mathrm{Na}^{+}$and $\mathrm{Cl}^{-}$ions $(0.15 \mathrm{M})$.

Following that, the solvated system was subjected to energy minimization of 50000 steps using the Steepest Descent Gradient Algorithm. The minimized system was subjected to position-restrained MD under NVT and NPT ensembles were carried out to maintain the constant temperature of $300 \mathrm{k}$ and a pressure of $1 \mathrm{~atm}$. After equilibration of the system with constant temperature and pressure, the production MD run was performed for $50 \mathrm{~ns}$ [19].

\section{Density Functional Theory (DFT) analysis}

DFT analysis was used to calculate the electronic features of the atom in the charge transfer reaction and to understand the detailed chemistry reactivity of fucoidan phytocompound which was carried out by Jaguar module (Schrödinger LLC, NY, USA). A whole geometrical optimization was performed using hybrid DFT with Berke's three-parameter exchange potential and the Lee-Yang-Parr correlation functional (B3LYP) and using the 3-21G* basis set. The Poisson-Boltzmann finite (PBF) solvation method was applied for energy calculations under aqueous environment. Molecular frontier orbital's like Highest Occupied Molecular Orbital (HOMO), Lowest Unoccupied Molecular Obital (LUMO) and its corresponding energy gap were calculated. Further, HOMO and LUMO distribution plot was visualized with Maestro panel in Schrödinger [20].

\section{ADME prediction}

Fucoidan phytocompound was tested for pharmaceutically relevant parameterss, drug likeness and pharmacokinetics properties such as Hydrigen bond acceptor, Hydrogen bond donor, Molecular weight, QPlogPo/w, QPPCaco, and \% of human oral absorption. All the properties were analusedusing Qikprop module of Schrödinger (Schrödinger LLC, NY, USA) [21].

\section{BOILED-Egg plot analysis}

The BOILED-Egg plot analysis is an accurate predictive model to calculate the gastrointestinal absorption and Blood Brain Barrier (BBB) penetrationof fucoidan phytocompound. It provides the special statistical plot to analyze the bioavailability characteristic of the fucoidan phytocompound. The analysis was carried out by using Swiss ADME online server [14].

\section{Results And Discussion}

\section{Molecular docking study}


In order to identify the potential drug candidate for managing dengue fever, Molecular Docking analysis was performed for Fucoidan against active site of DENVNS3 proteases of various DEN V Strains. This analysis provides to investigate the nature of binding mode between the protein-ligand complexes.Table 1 reveals the Docking Score ofFucoidan-DENV2 NS2B NS3 protease, Fucoidan-DENV3NS3 protease and FucoidanDENV4NS3 protease. From the table it is suggested thatthe fucoidan phytocompounds could be a potent lead compound with upon binding with target site of DENV NS3 proteases.

Table 1. Docking result of Fucoidan phytocompound against DENV NS3 proteases.

\begin{tabular}{|lllllll|}
\hline S.No & Target protein & $\begin{array}{l}\text { Compound } \\
\text { Name }\end{array}$ & $\begin{array}{l}\text { Docking Score } \\
\mathrm{kcal} / \mathrm{mol}\end{array}$ & $\begin{array}{l}\text { Glide } \\
\text { Energy } \\
\mathrm{kcal} / \mathrm{mol}\end{array}$ & $\begin{array}{l}\text { Glide } \\
\text { emodel } \\
\mathrm{kcal} / \mathrm{mol}\end{array}$ & $\begin{array}{c}\Delta \mathrm{G}_{\text {Bind }} \\
\mathrm{kcal} / \mathrm{mol}\end{array}$ \\
\hline 1 & $\begin{array}{l}\text { DENV2/NS2B NS3 } \\
\text { protease }\end{array}$ & & -6.458 & -32.287 & -48.241 & -41.25 \\
\hline 2 & \begin{tabular}{llllll|} 
DENV3NS3 protease \\
3
\end{tabular} & $\begin{array}{l}\text { DENV4 NS3 } \\
\text { protease }\end{array}$ & -6.987 & -39.891 & -41.311 & -44.38 \\
\hline
\end{tabular}

The fig. 1 (A) exhibits the 2D and 3D interactions diagram of fucoidan-DENV2/NS2B NS3 protease complex. The binding conformation forfucoidan-DENV2/NS2B NS3 protease complex showed docking score is -6.458 $\mathrm{kcal} / \mathrm{mol}$ and forms two hydrogen bonds with HIS51 and GLY151 amino acids with hydroxyl groups of fucoidan. The bond distances for His51 with $\mathrm{OH}$ and Gly 151 with $\mathrm{OH}$ are found to be for $2.58 \AA$ and $1.76 \AA$ respectively. In addition, numbers of hydrophobic interactions are formed With Met49, Val52, Asp75, Leu128, Phe130, Ser131, Pro132, Gly133, Thr134, Ser135, Tyr150, Asn152, Gly153, Val154, Tyr161, Val163 and Ser163 amino acid residues. The docking score for fucoidan-DENV3/NS3 protease complex is -6.987 $\mathrm{kcal} / \mathrm{mol}$ which has formedfour hydrogen bonds with Phe130 (bond distance $1.72 \AA$ ), Gly133 (bond distance $1.89 \AA$ ) , Thr134 (bond distance $2.73 \AA$ ) and Ser135 (bond distance $1.83 \AA$ ) with OH group. The complexis also attaching with other amino acids namely His51, Asp129, Lys131, Pro132, Thr150, Gly151 and Tyr161 are from hydrophobic interactions (Fig 1B). The binding mode of fucoidan-DENV4 NS3 protease complex exhibited a docking score of $-7.483 \mathrm{kcal} / \mathrm{mol}$ and hydroxyl group of fucoidan build a three hydrogen bonds with amino acids of Val36, His51 and Gly133. The bond distance between these complexes is found to be $2.01 \AA, 2.00 \AA$ and $2.26 \AA$ respectively. In addition, this fucoidan phytocompound are connected with six other amino acids such as GIn35, Gly37, Val52, Pro132, Ser135 and Asn152 by developing a hydrophobic interaction (Fig 1C).

The literature reports suggested that the NS3 protease is a trypsin-like serine protease which made up of $6 \beta$ strands that can forms two $\beta$-barrels with the catalytic triad of His51, Asp75 and Ser135 amino acids [22, 23]. There are two mechanisms involved for inhibition of protease; firstly ligand can either disturb electronic density or produce steric effect relating to the catalytic triad (His51, Asp75 and Ser135) amino acid residues. 
Second, it may disturb the movement of the C-terminal of the NS2B necessary for the transition between the "open" and "closed" formation of the protease [24]. In addition, interaction with one of the catalytic triad amino acid residues of protease can help to form the hydrogen bond which can disturb the electron transfer between the carboxyl groups containing Asp75 amino acid and nitrogen atom on the imidazole group containing His51. This happening leads to disruption of residue His51 ability to trigger the nucleophilic attack of the $\mathrm{OH}$ group of Ser135, basically required in the initiation of NS3 protease proteolytic activity [25]. In the present study, similar binding interaction with important catalytic triad of His51, Asp75 and Ser135 amino acids was observed and strongly formed hydrogen bond with $\mathrm{OH}$ group of fucoidan and hydrophobic interactions. The observed catalytic triad amino acids are actively participated with fucoidan phytocompound which may inhibit the protease activity. Therefore, fucoidan bioactive compound could be explored as a potential inhibitor for the further development of effective DENV drugs.

\section{Binding Free Energy Calculation}

Further, the MM/GBSA approach was applied to estimate the Binding Free Energy for fucoidan against NS3 proteases. A negative value of MM/GBSA suggested superior binding of the protein and ligand molecule. The Binding Free Energiesfor fucoidan with DENV2/NS2B NS3, DENV3 NS3 and DENV4 NS3 proteases complexes are found to be $41.25 \mathrm{kcal} / \mathrm{mol},-44.38 \mathrm{kcal} / \mathrm{mol}$ and $-47.22 \mathrm{kcal} / \mathrm{mol}$ respectively (Table 1). All the complexes exhibited better binding energy [26]. Therefore, the fucoidan phytocompound is deduced with substantiate binding affinity that can be strongly bound with catalytic triad of DENV NS3 proteases which may inhibit the viral proteolytic activity and prevent DENV fever.

\section{Molecular Dynamic Simulation}

Further, to substantiate the structure stability and conformational changes, MD simulation was performedfor docked complexes. Fig 2 (A-C) indicates the RMSD plot of Fucoidan-DENV2/NS2B NS3, Fucoidan-DENV3 NS3 and Fucoidan-DENV4 NS3 proteasecomplexes for 50 ns. Figure A shows the RMSD plot of backbone atoms and Fucoidan-DENV2/NS2B NS3 of protease complex. It seen that the average RMSD value for backbone (black colour) and complex (red colour) are $4.5 \pm 0.7$ and $5.3 \pm 0.85 \mathrm{~nm}$ respectively. In figure $\mathrm{B}$, during the simulation period, there is no significant change observed between backbone for DENV3 NS3 protease and Fucoidan-DENV3 NS3 protease complex except equilibration stage from 0-5 ns. The average RMSD value for backbone and fucoidan-DENV3 NS3 protease complex are found to be $0.35 \pm 0.5$ and $0.3 \pm$ $0.8 \mathrm{~nm}$ respectively. In the fucoidan-DENV4 NS3 protease complex, no significant structural changes are obtained and similar RMSD value is noticed $(0.45 \pm 1.0 \mathrm{~nm})$. The set of RMSD results indicated that the fucoidan phytocompound interacted with the all proteases are constant stable and no significant deviation observed whole simulation period. Thus, all the complexes are considered for further analysis.

Similar dynamic characteristics for the backbone atoms are also reflected by the RMSF in the MDS. To further assess the protein stability and to realize the effects of fucoidan phytocompound on the NS3 protease structure's flexibility, the mean per residue fluctuation of the protein backbone was determined using RMSF analysis for the period of 50ns. The RMSF of backbone atom of all the NS3 proteases indicated that the protein exhibited low fluctuation when binding of with fucoidan phytocompound. The average RMSF values for DENV2/NS2B NS3, DENV3 NS3 and DENV4 NS3 proteases are maintained at $0.24 \mathrm{~nm}, 0.20 \mathrm{~nm}$ 
and $0.32 \mathrm{~nm}$ respectively (Fig 3. A-C). In figure $3 \mathrm{~A}$, the following amino acid residues such as Leu18 (0.5 $\mathrm{nm})$, Glu19 (0.48 nm), Trp50 (0.4 nm), Pro138 (0.36 nm), Gln110 (0.35 nm), Lys128 (0.35 nm), Thr111 (0.34 nm), Lys112 $(0.34 \mathrm{~nm})$, and Ser163 $(0.34 \mathrm{~nm})$ are in high fluctuations. The fucoidan-DENV3 NS3 protease complex has shown higher fluctuation with the amino acid residues of Val4 $(0.76 \mathrm{~nm})$, Pro8 $(0.74 \mathrm{~nm})$, Ser9 (0.59nm), Pro10 (0.46nm), Thr120 (0.45nm), LYS157 (0.44nm) and Asn158 (0.43nm) (Figure 3 B). The fucoidan-DENV4 NS3 protease complex exhibited higher fluctuations with amino acid residues such as Ser19 (0.70 nm), Asp520 (0.61 nm) Lys117 (0.56nm), Leu119 (0. 56nm), Glu20 (0.54nm), Arg28 (0.54nm), Lys157 $(0.54 \mathrm{~nm})$, Ser159 $(0.52 \mathrm{~nm})$ and Lys104 $(0.51 \mathrm{~nm})$ (Figure 3C). From the RMSF plot it reveals that most of the amino acid residues are stable and high fluctuation of amino acid residues are lying in the loop region; those fluctuations did not affect the fucoidan-NS3 protease complexes.

\section{Hydrogen Bond analysis}

The Hydrogen Bond interaction analysis is the key tool to determining the molecular recognition and binding strength of the complexes. Fig4 (A-C) depicts the Hydrogen Bond interaction between the fucoidanDENV2/NS2B NS3, fucoidan-DENV3 NS3 and fucoidan-DENV4 NS3 protease complexes. During the simulation time, all the complexes have indicated the strong and constant binding stability due to the nonconformational changes observed. Therefore, the fucoidan phytocompound could be a potential candidate for inhibiting the NS3 proteases activity.

\section{DFT study}

The DFT analysis gives important information about the global and local indices of the fucoidan phytocompound which can helps to examine the chemical reactivity of the fucoidan phytocompound. According to the frontier molecular orbital theory, the electron donor and acceptor properties are determined. Fig5 depicts the HOMO and LUMO distribution plot of fucoidan phytocompound. The figure shows that the energy values of HOMO and LUMO are found to be $-0.249 \mathrm{eV}$ and $0.060 \mathrm{eV}$ respectively. In the fucoidan phytocompound, HOMO region is located in the (2S, 3S, 4S, 5R)-4, 5-dihydroxy-2-methyltetrahydro-2H-pyran$3-y l$ hydrogen sulfate group while the LUMO region is occupied in the (2S, 3S)-1, 3-dihydroxybutan-2-yl hydrogen sulfate group. Form the analysis in infers that the fucoidan phytocompound has high HOMO energy value when compared with LUMO value, suggesting that the phytocompound mainly involved in the nucleophilic reaction $[27,28]$. The HOMO-LUMO energy gap (HLG) is obtained at $-0.189 \mathrm{eV}$, confirming that the phytocompound may demonstrate a higher inhibitory activity against the DENV Infection.

\section{ADME properties}

This study evaluates the fucoidan phytocompound for pharmacological relevance to ensure the drug likeness and drug's pharmacokinetics properties [28]. Table 2 gives the ADME properties of the fucoidan phytocompound. The table divulges that all the pharmacokinetic properties for fucoidan has acceptable range due to obey the Lipinski's rule of five and no violation obtained. Further physicochemical descriptors including QPlogPo/w, QPlogS, QPlogHERG, QPlogBB, and \% of Human Oral Absorption are fall in the acceptable ADME range. The overall results reveal that the fucoidan phytocompound is obeying the drug- 
likeness potteries for human use. Therefore this phytocompound could be considered as a potential antiviral agent against DENV.

Table 2. ADME properties of the fucoidan compound.

\begin{tabular}{|lllllllll|}
\hline $\begin{array}{l}\text { S. } \\
\text { No }\end{array}$ & $\begin{array}{l}\text { Compound } \\
\text { Name }\end{array}$ & MW $^{1}$ & DonorHB $^{2}$ & AccepterHB $^{3}$ & QPlogPw $^{4}$ & QPlogs $^{5}$ & \%HOA $^{6}$ & $\begin{array}{l}\text { QPlog } \\
\text { HERG }^{7}\end{array}$ \\
\hline 1 & Fucoidan & 242.243 & 3.00 & 9.00 & 14.343 & -1.011 & 65.387 & -1.368 \\
\hline
\end{tabular}

\section{BOILED-Egg plot study}

For the drug discovery process, gastrointestinal absorption and BBB penetration play an imperative role. This plot helps in the computation of polarity and lipophilicity of fucoidan phytocompound. In the analysis, the white region is indicates the gastrointestinal tract and yellow region notices the high polarity of the BBB. In addition, the blue color indicator reveals that the compound is actively effluxes by P-glycoprotein $\left(\mathrm{PGP}^{+}\right)$, while the red color indicator exhibited the non substrate of $\mathrm{Pgp}\left(\mathrm{PGP}^{-}\right)$[30]. In the present analysis, the fucoidan phytocompound is located in the white region, suggesting that the fucoidan phytocompound has highly gastrointestinal absorption which is easily absorbed by the gastrointestinal tract and do not exhibit BBB permeation (Fig6).

\section{Conclusion}

For the drug designing and discovery process against DENV fever, fucoidan phytocompound was used and docked with NS3 proteases. In this study, Molecular Docking, Binding Free Energy, Molecular Dynamic Simulation, DFT, ADME prediction and BOILED-Egg plot analyses were performed. In the Molecular Docking study, all the docked complexes (fucoidan-DENV2/NS2B NS3 protease, fucoidan-DENV3 NS3 protease and fucoidan-DENV4 NS3 protease) showed good Docking score and actively interacted with catalytic triad of His51, Asp75 and Ser135 amino acids. Further good Binding Free Energy is obtained from Binding Free Energy calculation. The Molecular Dynamics Simulation study reveals that the fucoidan phytocompound interacted with the proteases from the entire DENV strains thatare constant stable and no significant deviation observed throughout simulation period. The DFT analysis reveals that the fucoidan phytocompound has high HOMO energy value when compared with LUMO value, suggesting that the phytocompound mainly involved in the nucleophilic reaction. The ADME prediction shows that the fucoidan phytocompound has obeyed all the pharmaceutical relevant parameters. Finally, the compound is highly absorbed by the gastrointestinal tract which was evaluated by BOILED-Egg plot analysis. Based on the outcome of the results from in silico analysis, we suggested that the fucoidan polysaccharide from Padinagymnospora could be a promising lead compound as antiviral agent targeting DEN $V$ structural protein. 


\section{Declarations}

\section{Funding}

The authors thankfully acknowledge the UGC-Innovative [No.F. 14-13/2013 (Inno/ASIST)], DST-FIST [SR/FST/LSI-667/2016(C)], DST PURSE [SR/PURSE Phase 2/38 (G)], MHRD-RUSA 2.0 [F.24/51/2014-U Policy (TNMulti-Gen), Dept. of Edn. Govt. of India] and DST-SERB [EEQ/2016/000044] for the financial supports and infrastructure facilities.

\section{Authors' contributions}

Ramalingam Swaminathan: Contributed to design and implemented of the work

Sankar Muthumanickam: Investigation, Methodology, Writing - original draft

Venkataraman Anuradha: Contributed to the final version of the manuscript

Mohamed yacoob Syed Ali: Writing - review \& editing, Supervision

Pandi Boomi Conceptualization, Methodology, Writing - review \& editing, Supervision

Balajee Ramachandran: Review \& editing \& designed the figure

Availability of data and material: The original contributions presented in the study are included in the article; further inquiries can be directed to the corresponding author/s.

\section{Conflicts of interest}

There are no conflicts to declare.

\section{References}

1. Murray NEA, Quam MB, Wilder-Smith A (2013) Epidemiology of dengue: Past, present and future prospects. Clin Epidemiol 5:299-309. https://doi.org/10.2147/CLEP.S34440

2. Lin K-H, Ali A, Rusere L et al (2017) Dengue Virus NS2B/NS3 Protease Inhibitors Exploiting the Prime Side. J Virol 91. https://doi.org/10.1128/jvi.00045-17

3. Lim SYM, Chieng JY, Pan Y (2021) Recent insights on anti-dengue virus (DENV) medicinal plants: review on in vitro, in vivo and in silico discoveries. All Life 14:1-33.

https://doi.org/10.1080/26895293.2020.1856192

4. Thisyakorn U, Thisyakorn C (2014) Latest developments and future directions in dengue vaccines. Therapeutic Adv Vaccines 2:3-9. https://doi.org/10.1177/2051013613507862

5. Davidson RB, Hendrix J, Geiss BJ, McCullagh M (2018) Allostery in the dengue virus NS3 helicase: Insights into the NTPase cycle from molecular simulations. PLoS Comput Biol 14:1-28. https://doi.org/10.1371/journal.pcbi.1006103 
6. Niyomrattanakit P, Winoyanuwattikun P, Chanprapaph S et al (2004) Identification of Residues in the Dengue Virus Type 2 NS2B Cofactor That Are Critical for NS3 Protease Activation. J Virol 78:1370813716. https://doi.org/10.1128/jvi.78.24.13708-13716.2004

7. Wu DW, Mao F, Ye Y et al (2015) Policresulen, a novel NS2B/NS3 protease inhibitor, effectively inhibits the replication of DENV2 virus in BHK-21 cells. Acta Pharmacol Sin 36:1126-1136. https://doi.org/10.1038/aps.2015.56

8. Silva EM, Conde JN, Allonso D et al (2019) Dengue virus nonstructural 3 protein interacts directly with human glyceraldehyde-3-phosphate dehydrogenase (GAPDH) and reduces its glycolytic activity. Sci Rep 9:1-20. https://doi.org/10.1038/s41598-019-39157-7

9. Abd Kadir SL, Yaakob H, Mohamed Zulkifli R (2013) Potential anti-dengue medicinal plants: A review. J Nat Med 67:677-689. https://doi.org/10.1007/s11418-013-0767-y

10. Yokota T, Nomura K, Nagashima M, Kamimura N (2016) Fucoidan alleviates high-fat diet-induced dyslipidemia and atherosclerosis in ApoEshl mice deficient in apolipoprotein E expression. J Nutr Biochem 32:46-54. https://doi.org/10.1016/j.jnutbio.2016.01.011

11. Elizondo-Gonzalez R, Cruz-Suarez LE, Ricque-Marie D et al (2012) In vitro characterization of the antiviral activity of fucoidan from Cladosiphon okamuranus against Newcastle Disease Virus. Virol J 9:1-9. https://doi.org/10.1186/1743-422X-9-307

12. Synytsya A, Bleha R, Synytsya A et al (2014) Mekabu fucoidan: Structural complexity and defensive effects against avian influenza A viruses. Carbohydr Polym 111:633-644. https://doi.org/10.1016/j.carbpol.2014.05.032

13. Anand K, Naicker T, Baijnath S et al (2020) TPGS-mediated one-pot synthesis, XRD structural analysis, antimicrobial evaluation and molecular docking of novel heterocycles as potential inhibitors of p53MDM2 protein. J Mol Struct 1202:127252. https://doi.org/10.1016/j.molstruc.2019.127252

14. Muthumanickam S, Indhumathi T, Boomi P et al (2020) In silico approach of naringin as potent phosphatase and tensin homolog (PTEN) protein agonist against prostate cancer. J Biomol Struct Dynamics 0:1-10. https://doi.org/10.1080/07391102.2020.1830855

15. Ramachandran B, Srinivasadesikan V, Chou TM et al (2020) Atomistic simulation on flavonoids derivatives as potential inhibitors of bacterial gyrase of Staphylococcus aureus. J Biomol Struct Dynamics 0:1-14. https://doi.org/10.1080/07391102.2020.1856184

16. Sankar M, Ramachandran B, Pandi B et al (2021) In silico Screening of Natural Phytocompounds Towards Identification of Potential Lead Compounds to Treat COVID-19. Front Mol Biosci 8. https://doi.org/10.3389/fmolb.2021.637122

17. Mejia-Gutierrez M, Vásquez-Paz BD, Fierro L, Maza JR (2021) In Silico Repositioning of Dopamine Modulators with Possible Application to Schizophrenia: Pharmacophore Mapping, Molecular Docking and Molecular Dynamics Analysis. ACS Omega 6:14748-14764. https://doi.org/10.1021/acsomega.0c05984

18. Gowrishankar S, Muthumanickam S, Kamaladevi A et al (2021) Promising phytochemicals of traditional Indian herbal steam inhalation therapy to combat COVID-19 - An in silico study. Food Chem Toxicol 148:111966. https://doi.org/10.1016/j.fct.2020.111966 
19. Muthumanickam S, Kamaladevi A, Boomi P et al (2021) Indian Ethnomedicinal Phytochemicals as Promising Inhibitors of RNA-Binding Domain of SARS-CoV-2 Nucleocapsid Phosphoprotein: An In Silico Study. Front Mol Biosci 8. https://doi.org/10.3389/fmolb.2021.637329

20. Choubey SK, Jeyaraman J (2016) A mechanistic approach to explore novel HDAC1 inhibitor using pharmacophore modeling, 3D- QSAR analysis, molecular docking, density functional and molecular dynamics simulation study. J Mol Graph Model 70:54-69. https://doi.org/10.1016/j.jmgm.2016.09.008

21. Sankar M, Jeyachandran KL, Pandi S B (2021) Screening of inhibitors as potential remedial against Ebolavirus infection: pharmacophore-based approach. J Biomol Struct Dynamics 39:395-408. https://doi.org/10.1080/07391102.2020.1715260

22. Lim L, Dang M, Roy A et al (2020) Curcumin Allosterically Inhibits the Dengue NS2B-NS3 Protease by Disrupting Its Active Conformation. ACS Omega 5:25677-25686. https://doi.org/10.1021/acsomega.0c00039

23. Dwivedi VD, Tripathi IP, Bharadwaj S et al (2016) Identification of new potent inhibitors of dengue virus NS3 protease from traditional Chinese medicine database. VirusDisease 27:220-225. https://doi.org/10.1007/s13337-016-0328-6

24. Sriram Uday RV, Misra R, Harika A et al (2021) Dabrafenib, idelalisib and nintedanib act as significant allosteric modulator for dengue NS3 protease. PLoS ONE 16:1-20. https://doi.org/10.1371/journal.pone.0257206

25. Dwivedi VD, Bharadwaj S, Afroz S et al (2021) Anti-dengue infectivity evaluation of bioflavonoid from Azadirachta indica by dengue virus serine protease inhibition. J Biomol Struct Dynamics 39:1417-1430. https://doi.org/10.1080/07391102.2020.1734485

26. Bharadwaj S, Lee KE, Dwivedi VD et al (2019) Discovery of Ganoderma lucidum triterpenoids as potential inhibitors against Dengue virus NS2B-NS3 protease. Sci Rep 9:1-12. https://doi.org/10.1038/s41598-019-55723-5

27. Mariadasse R, Choubey SK, Jeyakanthan J (2020) Insights into Exogenous Tryptophan-Mediated Allosteric Communication and Helical Transition of TRP Protein for Transcription Regulation. J Chem Inf Model 60:175-191. https://doi.org/10.1021/acs.jcim.9b00755

28. Choubey SK, Richard M, Santhosh R, Jeyakanthan J (2016) Identification of novel Histone Deacetylase 1 inhibitors by combined PharmacophoreModeling, 3D-QSAR analysis, In Silico Screening and Density Functional Theory (DFT) approaches. J Mol Struct 391-404. DOI: 10.1016/j.molstruc.2016.06.082

29. Prajisha J, Biswal J, Jeyakanthan J (2020) Discovery of potent Camkk1 kinase inhibitors through epharmacophore and molecular screening approaches. J Biomol Struct Dynamics 0:1-17. https://doi.org/10.1080/07391102.2020.1842805

30. Zafar F, Gupta A, Thangavel K et al (2020) Physicochemical and Pharmacokinetic Analysis of Anacardic Acid Derivatives. ACS Omega 5:6021-6030. https://doi.org/10.1021/acsomega.9b04398

\section{Figures}




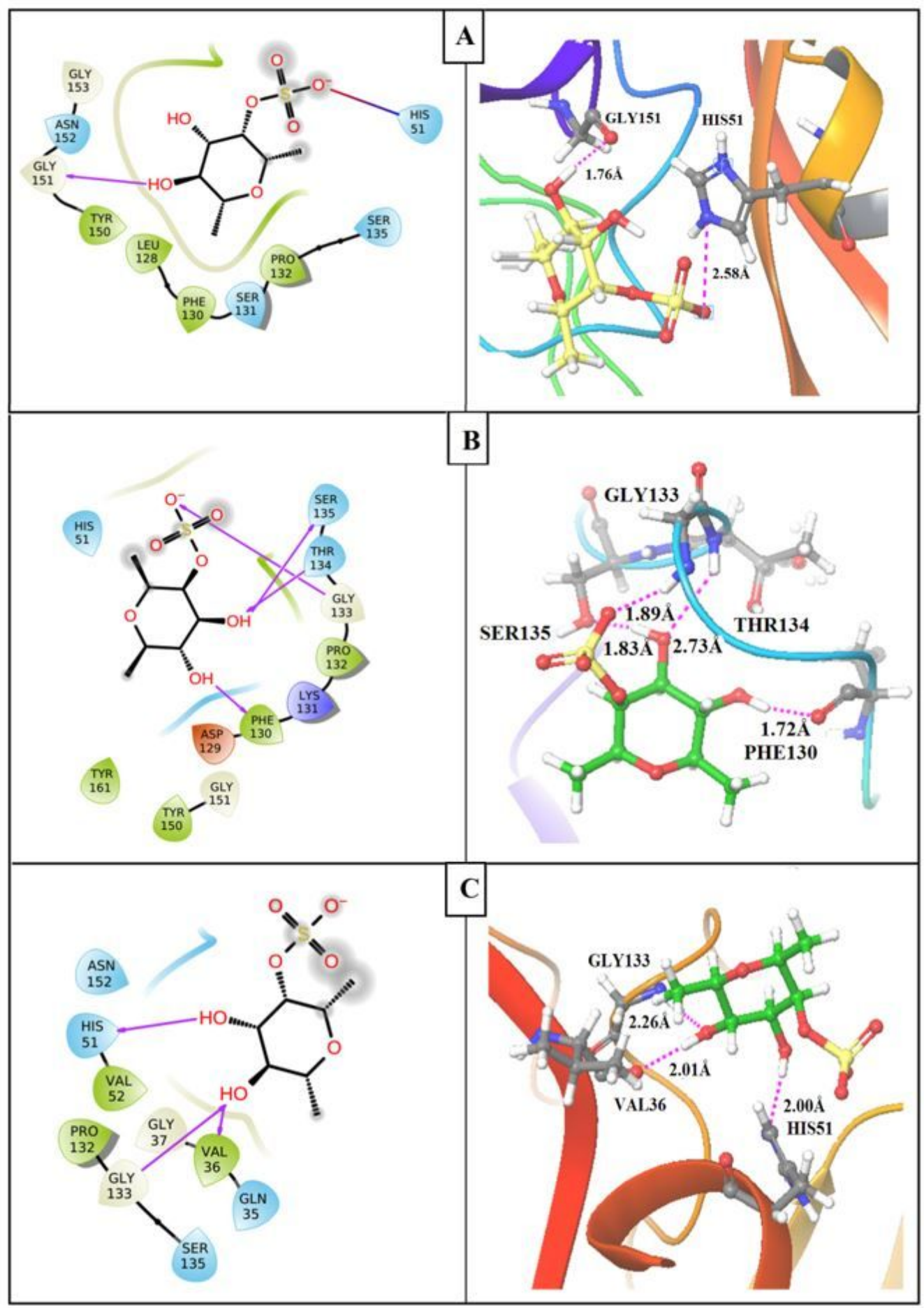

Figure 1

$2 \mathrm{D}$ and $3 \mathrm{D}$ diagram of Fucoidan-DENV2/NS2B NS3 protease complex (A), $2 \mathrm{D}$ and $3 \mathrm{D}$ diagram of Fucoidan-DENV2 NS3 protease complex (B) and $2 \mathrm{D}$ and $3 \mathrm{D}$ diagram of Fucoidan-DENV2 NS3 protease complex (C). 


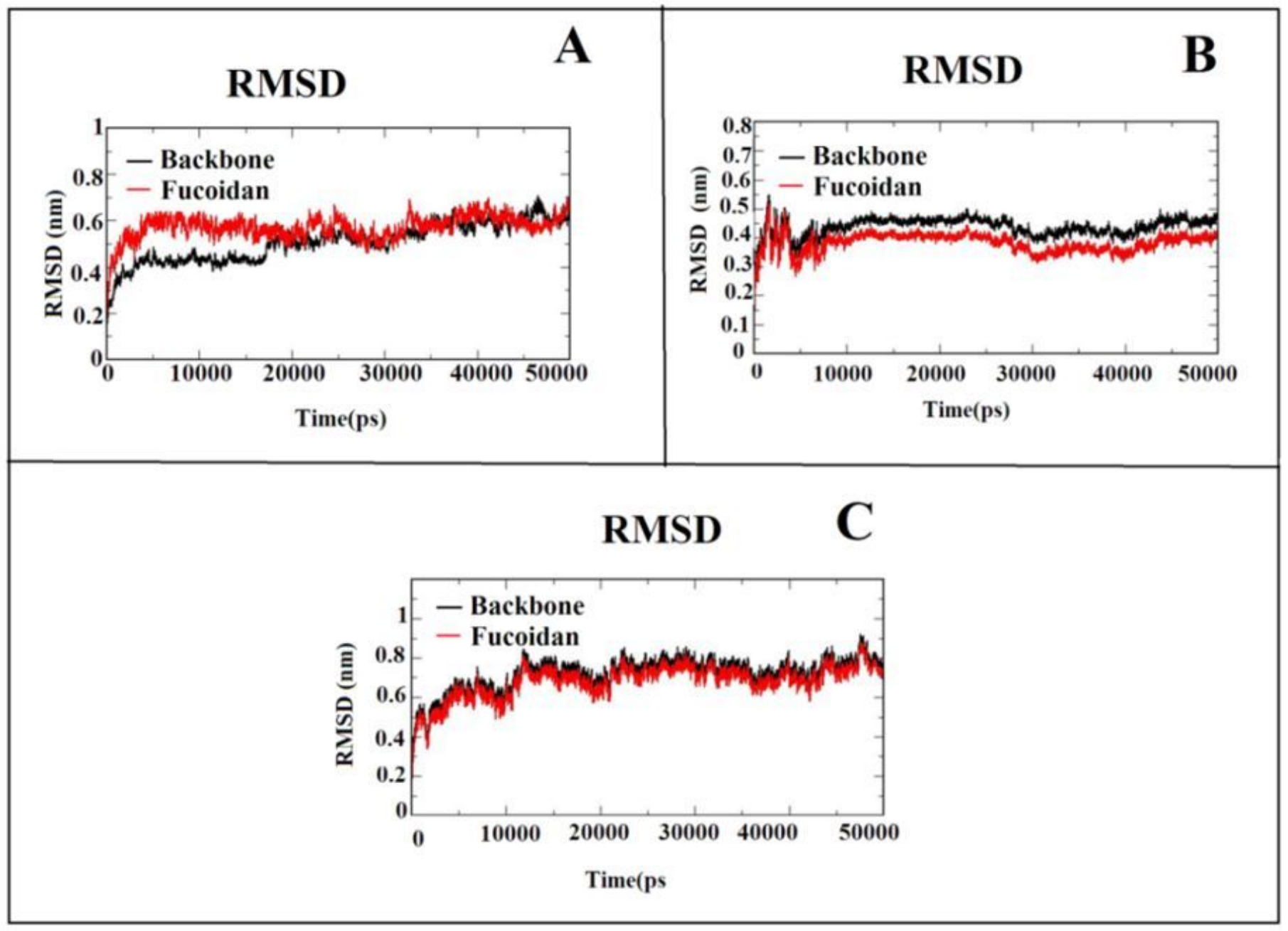

Figure 2

RMSD Plot of all protein-ligand complexes as Functional time period. A) Fucoidan with DENV2 NS2B/NS3 protease, B) Fucoidan with DENV3 NS3 protease, C) Fucoidan with DENV4 NS3 protease. 


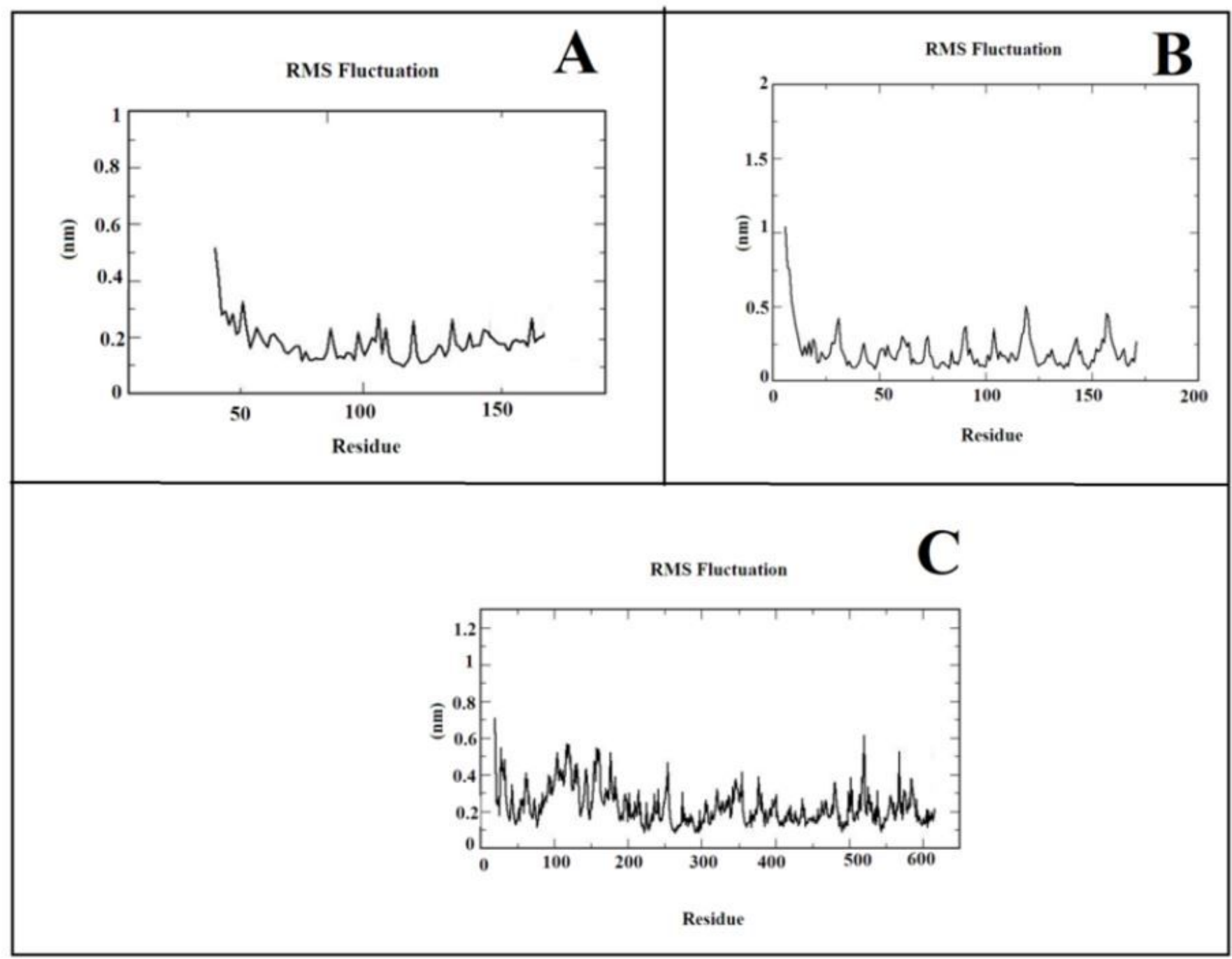

Figure 3

RMSF Plot of (A) fucoidan-DENV2/NS2B NS3 protease, (B) fucoidan-DENV3 NS3 protease and(C) fucoidanDENV4 NS3 protease. 


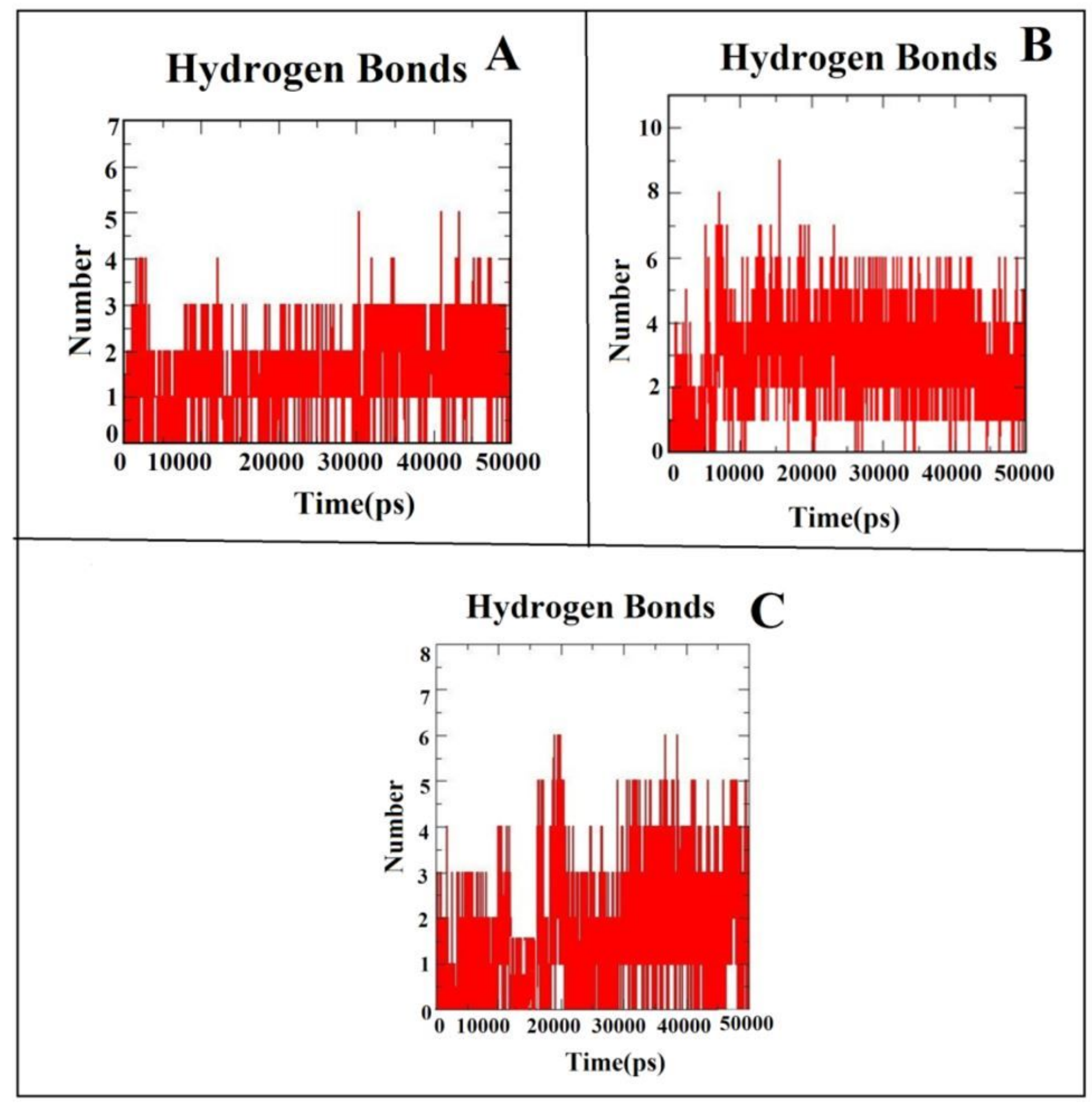

Figure 4

Hydrogen Bond analyses of (A) fucoidan-DENV2/NS2B NS3 protease, (B) fucoidan-DENV3 NS3 protease and (C) fucoidan-DENV4 NS3 protease. 


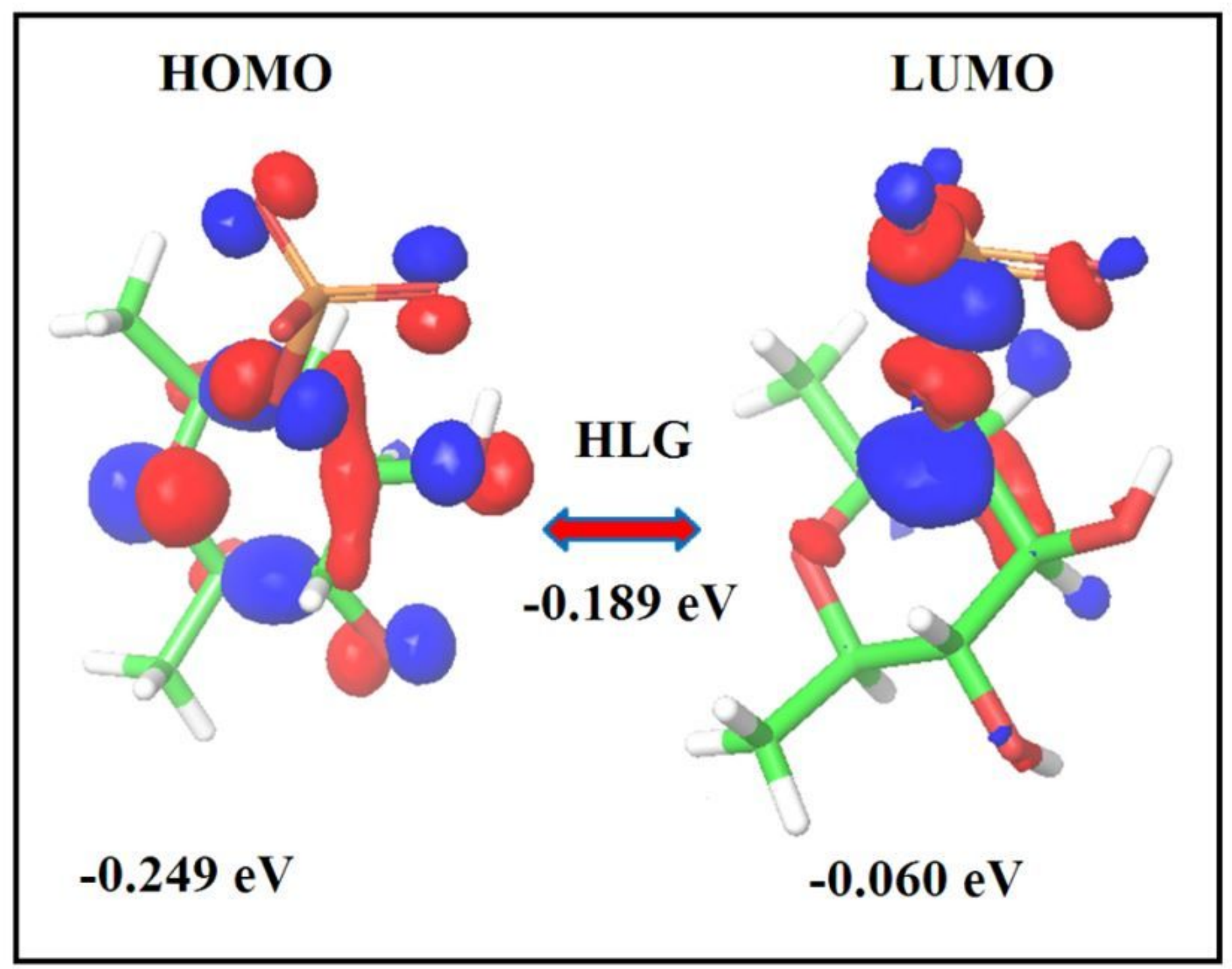

Figure 5

HOMO and LUMO distribution plot of fucoidan phytocompound 


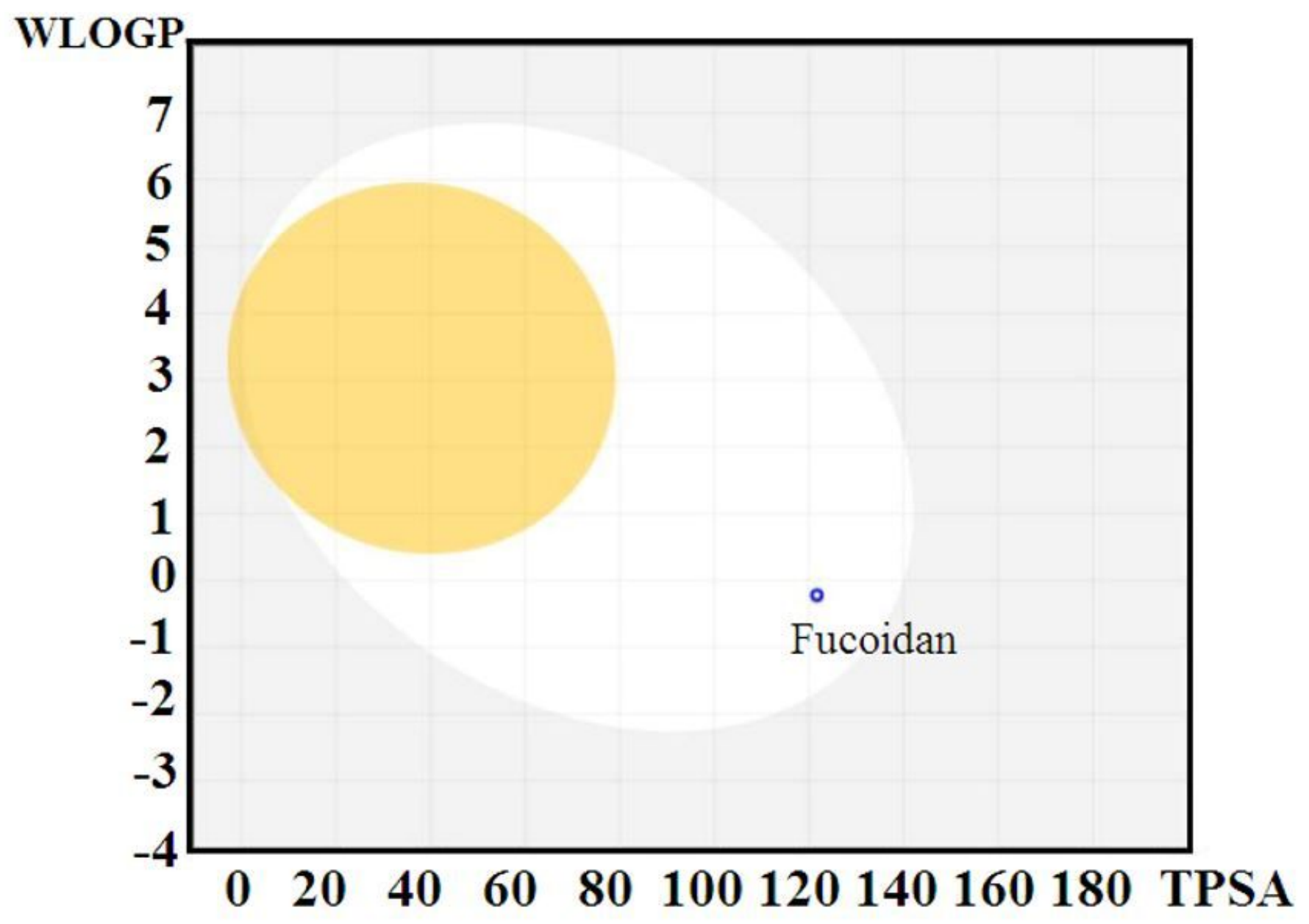

Figure 6

BOILED-Egg plot analysis of fucoidan phytocompound

\section{Supplementary Files}

This is a list of supplementary files associated with this preprint. Click to download.

- Graphicalabstract.tif 